\title{
PULMONARY TUBERCULOSIS AND PREGNANCY
}

\author{
By JOSEPH SMART, M.D., M.R.C.P. \\ (Physician in Charge Out-Patients, The London Chest Hospital; Casualty Physician, St. Bartholomew's \\ Hospital; Physician to an E.M.S. Hospital)
}

\section{Introduction.}

It is well known that in most cases of active or quiescent pulmonary tuberculosis pregnancy has a detrimental effect on the lung condition. Generally this does not become apparent during pregnancy, but in the months following parturition. In cases of healed pulmonary tuberculosis there is very little risk of the disease breaking down following pregnancy, but the difficulty which faces the clinician is that of determining for certain when the condition has become healed and not merely quiescent.

There are several factors to account for the phenomena that during pregnancy the patient remains well, and often feels much better than before. During the early stages of pregnancy there is very little extra strain on the patient because the amount of energy that has to be supplied by the potential mother is slight. At the same time the patient has the great psychological satisfaction of being pregnant which makes her very contented; therefore she has the mental peace, with little extra physical exercise, which is good for her. During the later months of pregnancy there are two factors which account for her well-being: (i) the patient's physical activities are automatically reduced as the size of the foetus increases, and (ii) the diaphragms are pushed up by the increased abdominal pressure, thereby relaxing the lungs considerably. At the time of parturition there is a great expenditure of energy; followed by a sudden relaxation of the diaphragms, and, unless the patient is carefully instructed, she soon returns to normal life, with increased activity because she has to look after and feed her baby, return to her household duties, and to all this is added the strain of disturbed nights. The result is that in the months immediately following the birth of the child the tuberculous disease in the lungs is liable to break down, or it may spread.

\section{Active Pulmonary Tuberculosis and Pregnancy}

In a case of active pulmonary tuberculosis the pregnancy, as a general rule, should be terminated. Should the patient, however, be four or more months pregnant before seeking medical advice, then it is better for the pregnancy to continue to full term.

The reason for allowing the continuance of a pregnancy after the fourth month is that termination at this stage would involve an abdominal operation, i.e. a hysterotomy, and experience has shown that, following such a procedure, the patient is just as liable to have a breakdown with a spread of the disease as she is if the pregnancy is allowed to continue. During the first three months the pregnancy can be terminated per vaginum with much less disturbance to the patient.

During recent years it has been shown that in cases of active pulmonary tuberculosis pregnancy can be allowed to continue with relative safety provided that the patient is willing to rest sufficiently not only during pregnancy, but also after parturition, and that in this way the disease can be controlled; collapse therapy in cases of unilateral disease, when possible, will further reduce the risk to the mother.

Cohen (I943) has shown that in cases of active pulmonary tuberculosis pregnancy does not cause a marked increase in the disease present provided the patient is under strict supervision during the pregnancy, labour, and for some months after the birth of the child. In Ioo cases of pulmonary tuberculosis complicated by pregnancy and treated at a sanatorium where a labour ward was available, only I2 showed signs of retrogression, 5 of which improved with sanatorium treatment. Of these cases 25 had active disease, of which 7 showed signs of retrogression, and 2 of these improved after sanatorium treatment. Of the others, 46 were arrested and 29 quiescent cases. Three of the former broke down, of which 2 improved later; of the 29 quiescent cases, 2 broke down, one of which improved later. This experiment followed the publication of some results in America in which it was stated that, with adequate sanatorium treatment before and after the pregnancy, the disease did not progress. Cohen's results support this view, because in a certain number of cases without the complication of pregnancy, even 


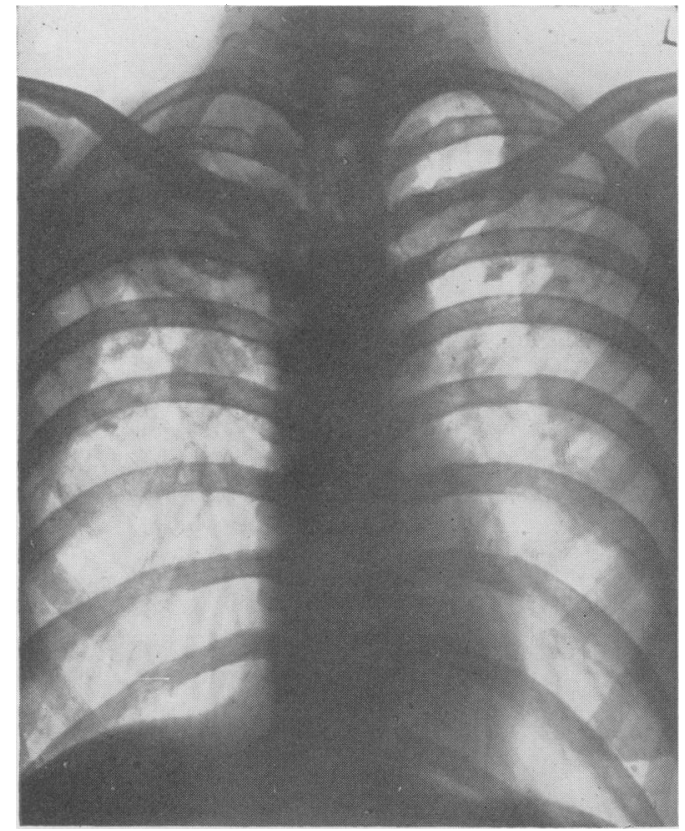

FIG. IA, Mrs. S., I. Io.43.-Bilateral active pulmonary tuberculosis.

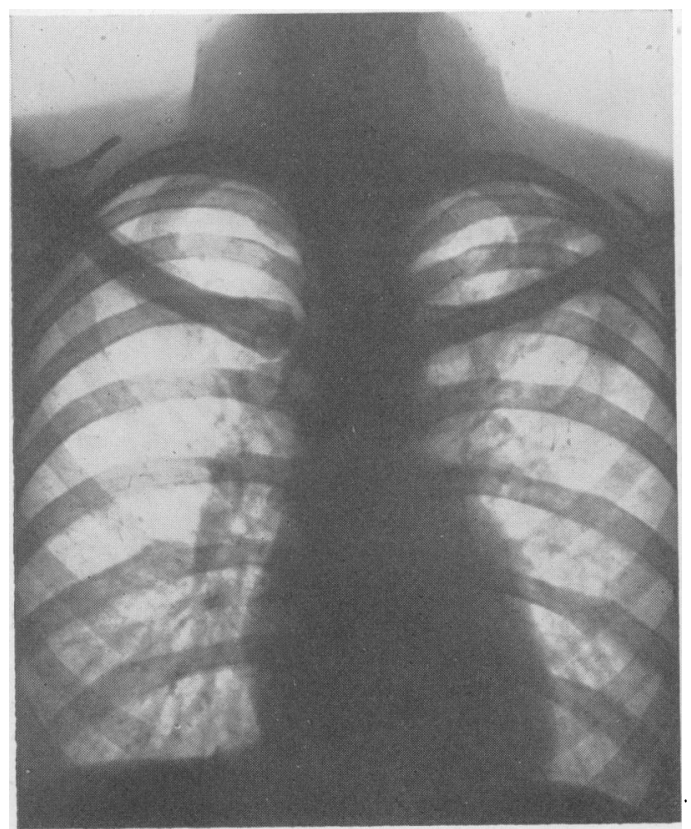

FIG. 2A, Mrs. M., I.5.4I.-Left upper lobe pulmonary tuberculosis with cavitation. Six months pregnant.

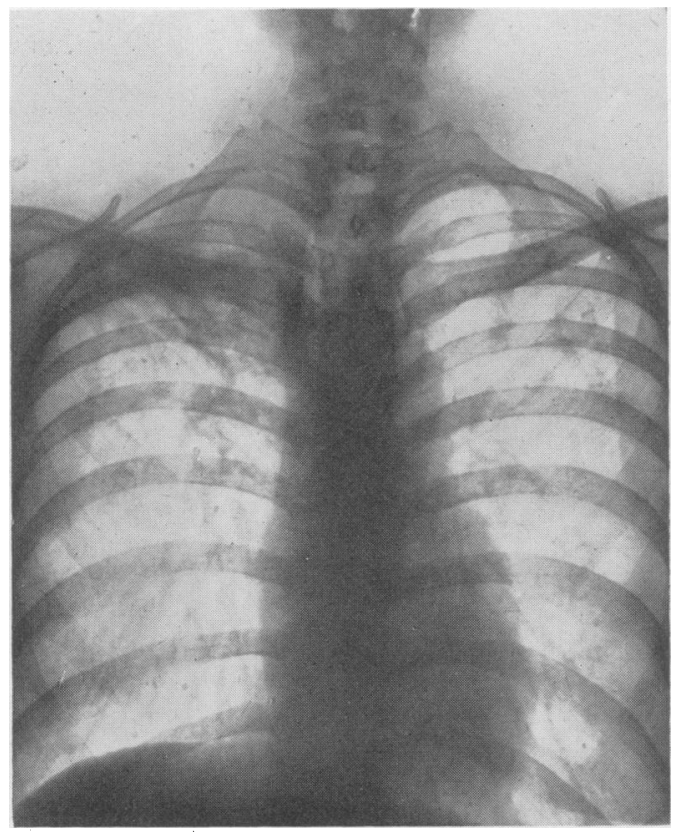

FIG. IB, Mrs. S., I6.2.44--Following termination of pregnancy and rest.

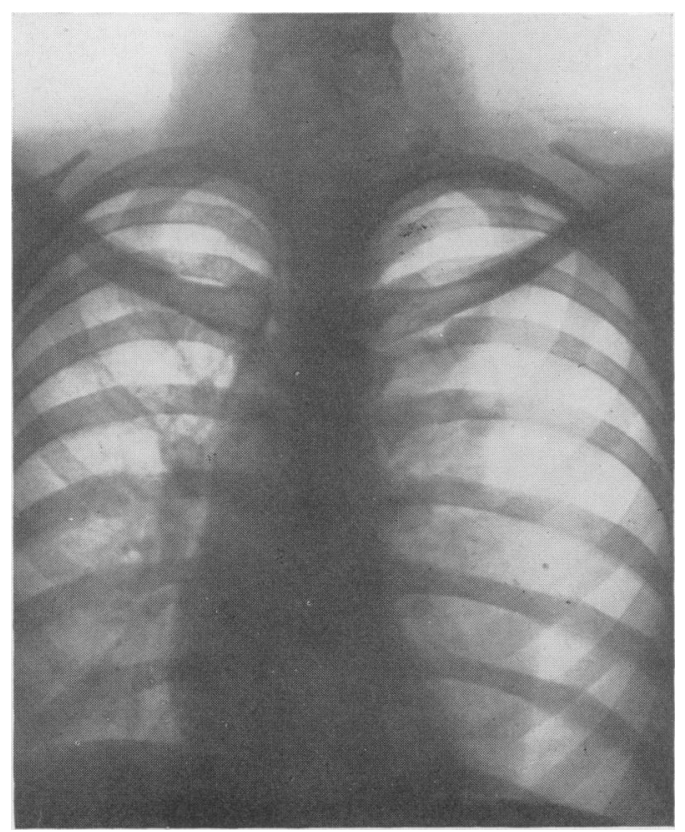

FIG. 2B, Mrs. M., I 5.5.4I.-Two weeks later following induction of left A.P. Pregnancy continued. 


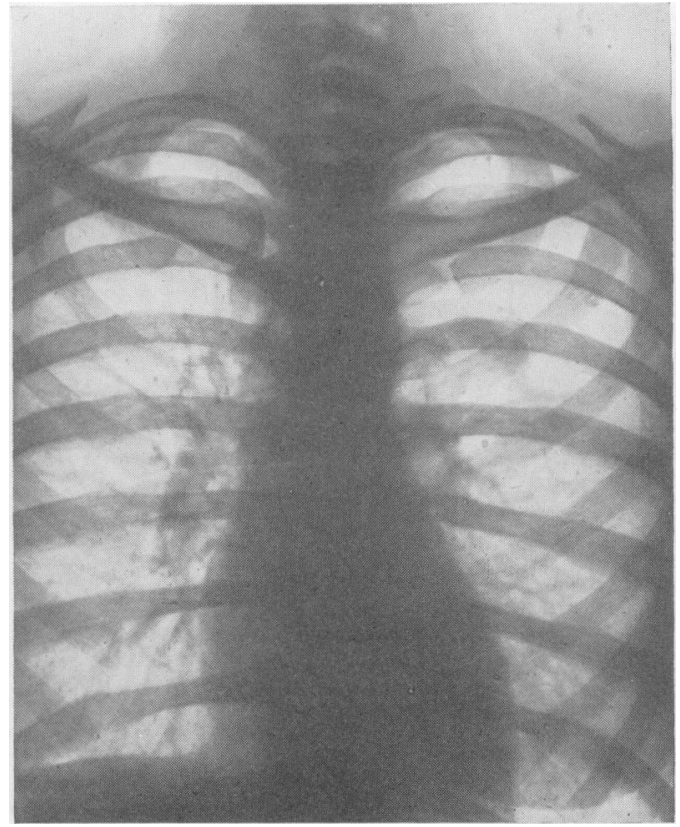

FIG. 2C, Mrs. M., I 2.6.4I.-Following thorasoscopy and complete freeing of left lung. Cavity still visible.

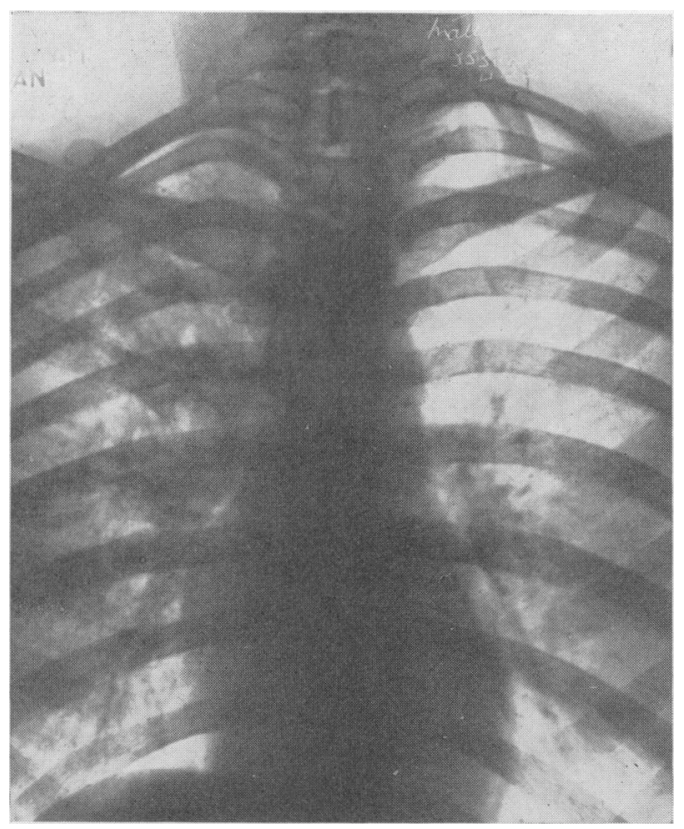

FIG. 3A, Mrs. B, 4.2.35.-Active pulmonary tuberculosis left. Shadowing over most of lung field with cavity close to hilum.

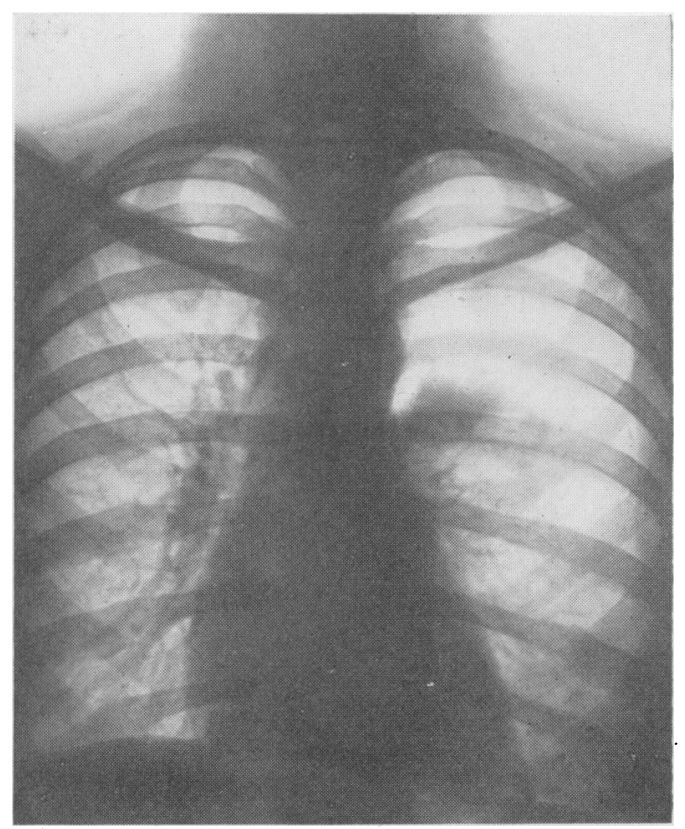

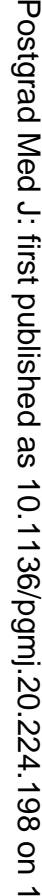

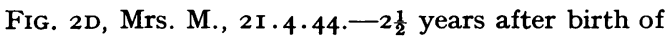
the child. A.P. continuing.

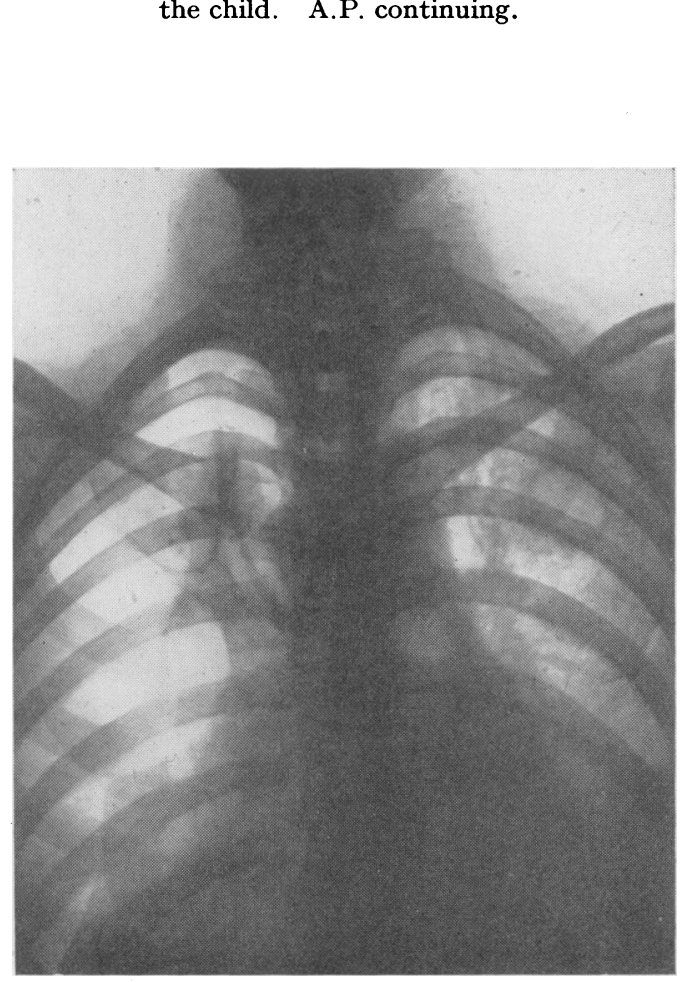

FIG. 3B, Mrs. B., 24.5 36.-A.P. right, with

adhesions. Large cavity left sub-apical region. 


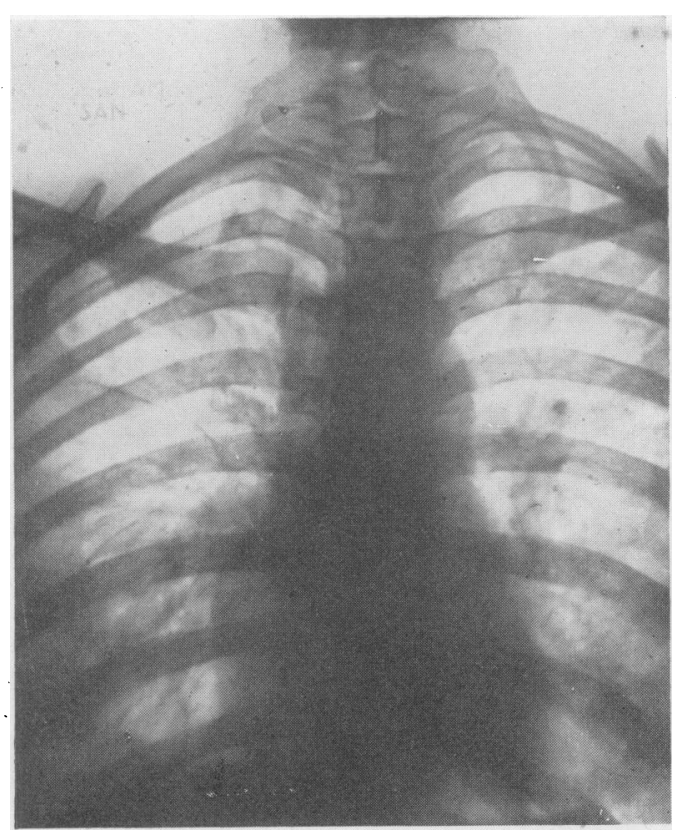

FIG. 3c, Mrs. B., I5.4.37.-Right lung re-expanded. Cavity probably still present in left sub-apical region.

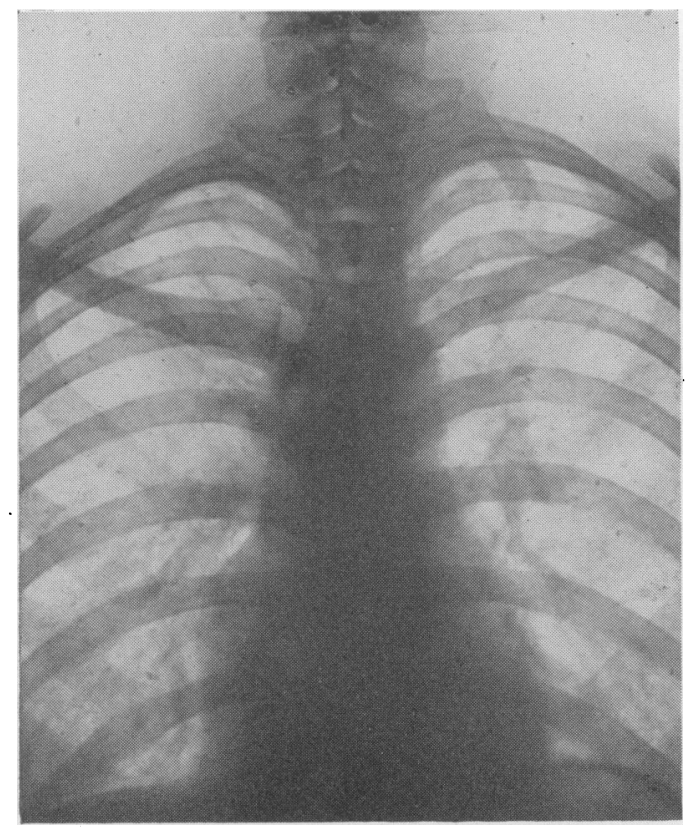

FIG. 3E, Mrs. B., 6.8.42.- Scarring and fibrosis on the left side. Disease now regarded as quiescent and pregnancy allowed.

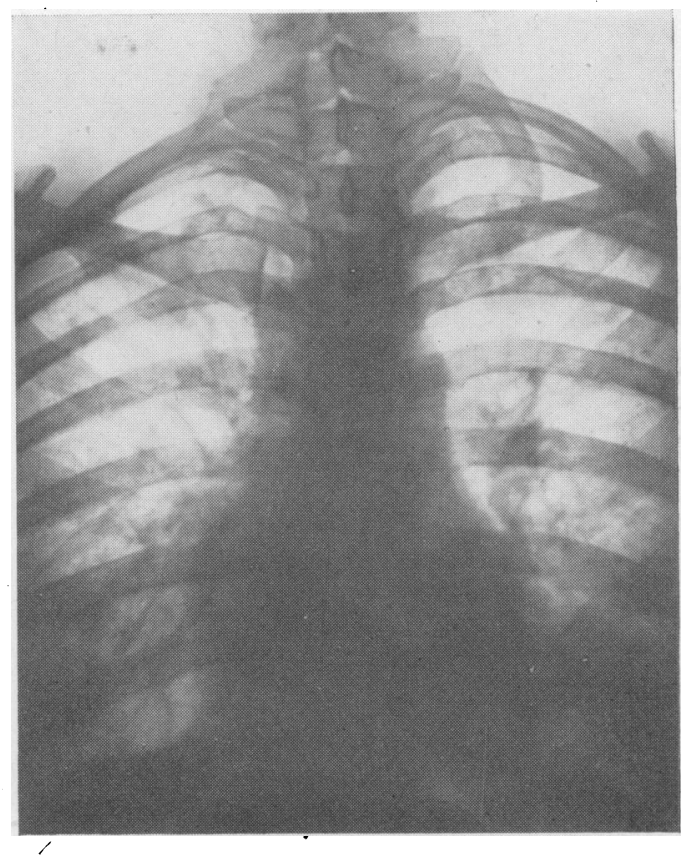

Fig. 3D, Mrs. B, 20.I.38.-Infiltration right subapical region. Cavity on left side closed.

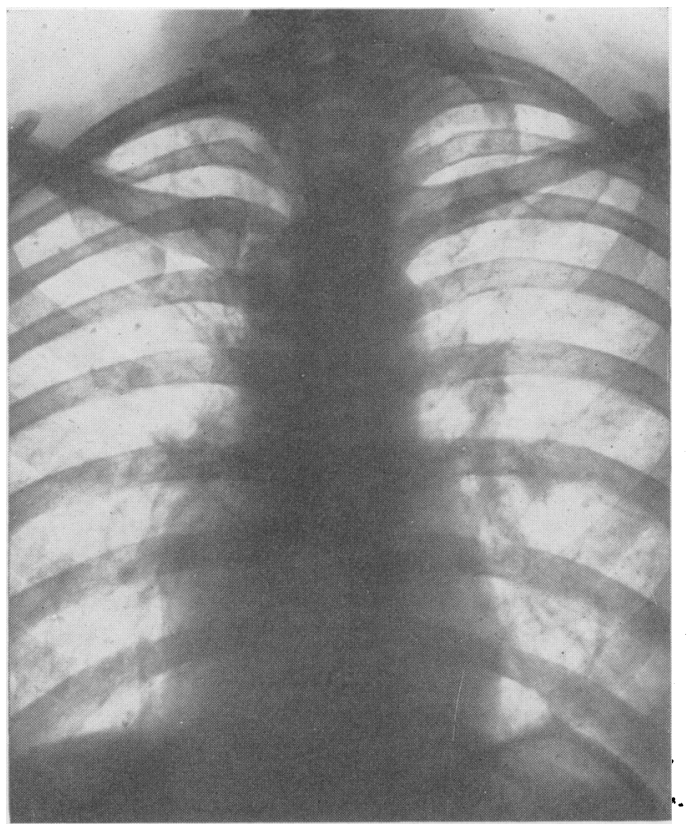

FIG. 3F, Mrs. B., I 7 5.44.-Fibrosis at both apices. No change from previous film. Ten months after birth of baby. 


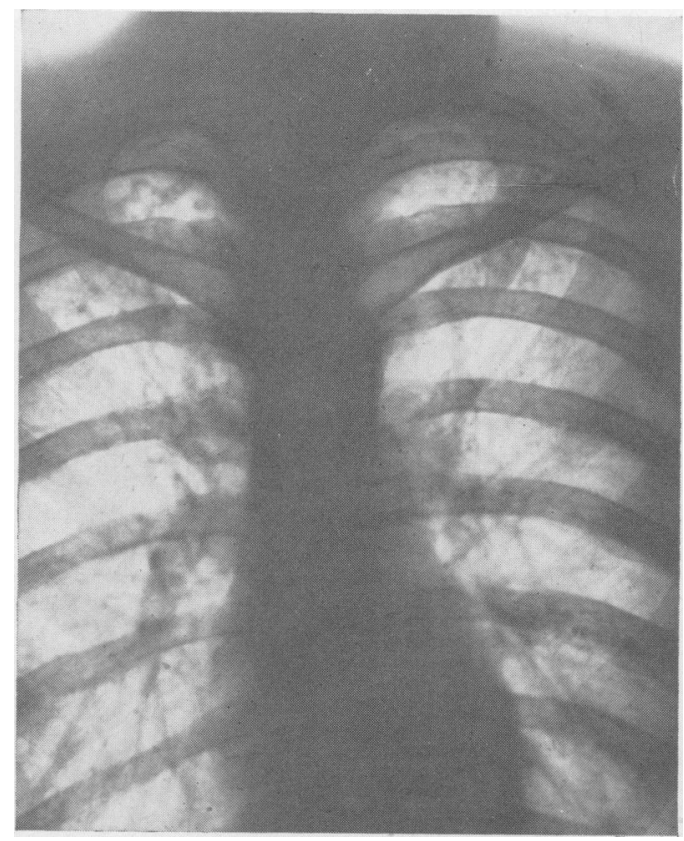

FIG. 4A, Mrs. A., 13.10.32.-Bilateral apical pulmonary tuberculosis.

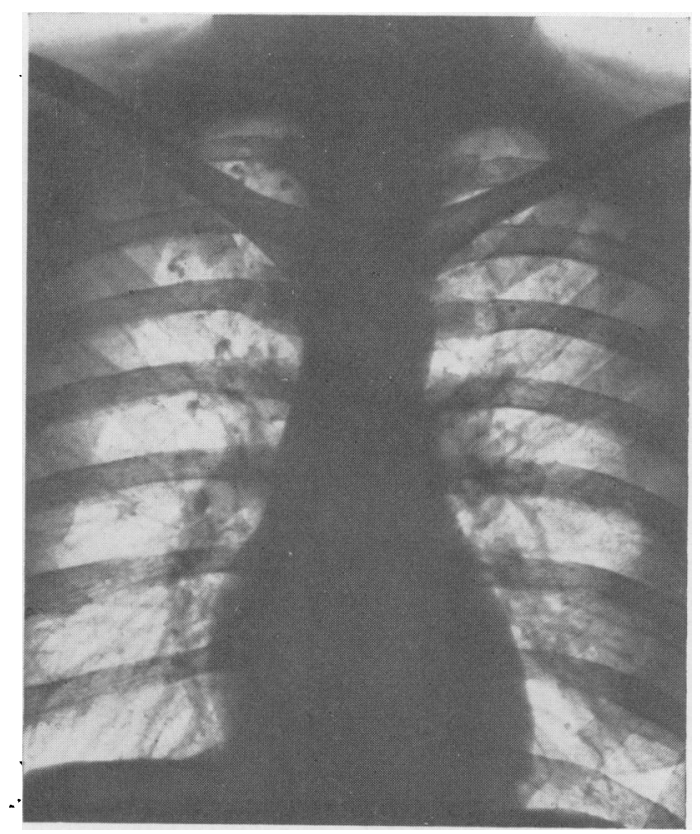

FIG. 4C, Mrs. A., 30.10.42.- Healed bilateral pulmonary tuberculosis, at full term.

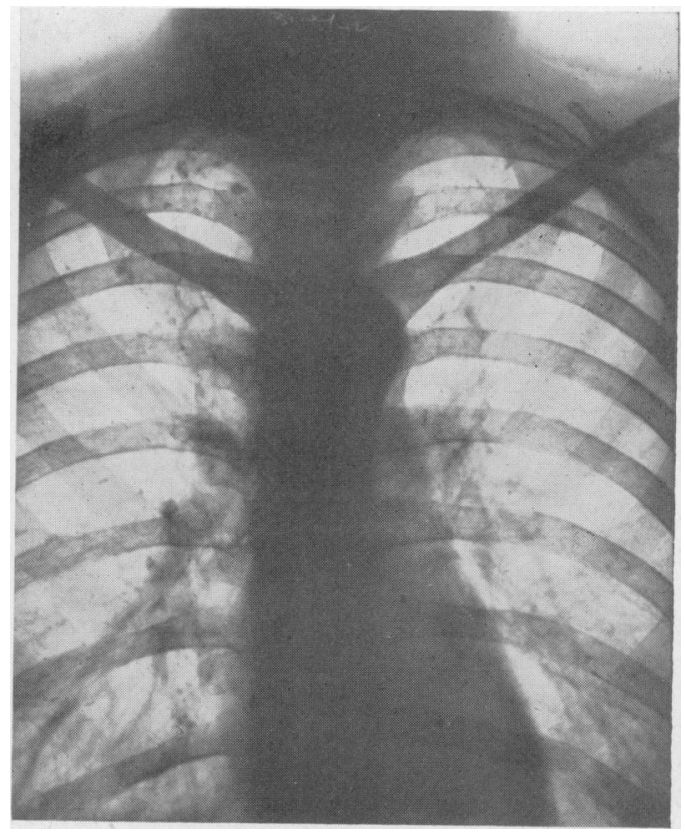

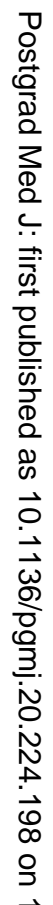

Fig. 4B, Mrs. A., 23.I.42.- Healed bilateral pulmonary tuberculosis.

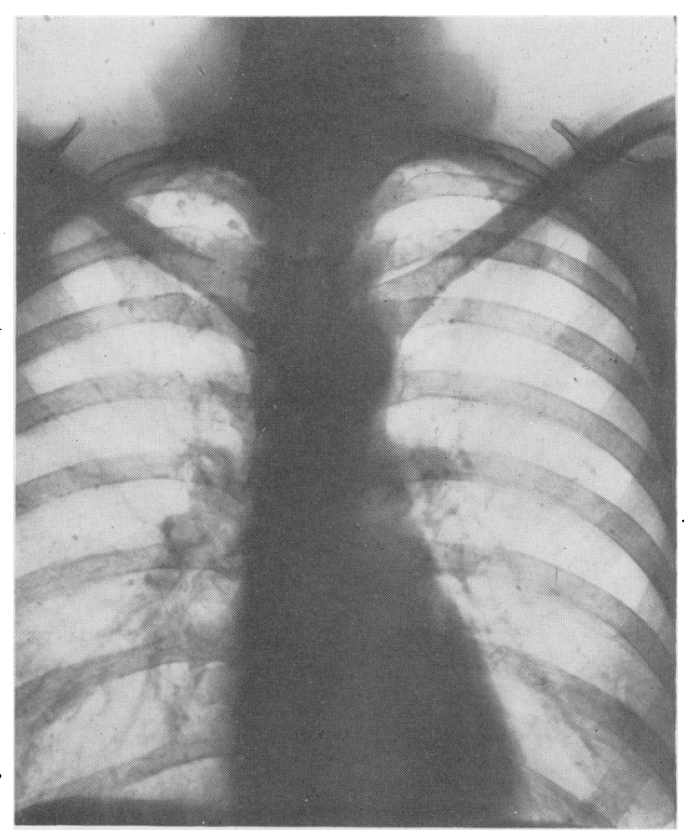

FIG. 4D, Mrs. A., 15.12.43.- Healed bilateral pulmonary tuberculosis just over one year after birth of child. 
with the best modern treatment, the disease is liable to pursue a progressive and relentless course. Cohen's view is not widely accepted in this country, but in my experience it is correct.

Pregnancy involves little risk to a patient with active unilateral disease, even if there is a positive sputum and cavitation, provided the disease can be adequately controlled by a pneumothorax, and the patient is willing to rest. To obtain adequate control of the disease it may be necessary not only to induce an artificial pneumothorax, but also to divide any adhesions present. Pregnancy is not a contraindication to adhesion section. The fact that the patient has a pneumothorax does not appear to cause any undue disturbance at the time of labour, and, although it is wise to have oxygen available at this time, it is seldom necessary. A refill should be given within a few hours of the birth of the child; otherwise the pressure in the pneumothorax is reduced considerably because of the sudden relaxation of the diaphragms. If the patient is not prepared to rest, and if an artificial pneumothorax cannot be obtained, then the pregnancy should be terminated.

In a case of active bilateral pulmonary tuberculosis when the patient is willing to rest as much as is necessary, and the pregnancy is allowed to continue, the induction of a pneumoperitoneum within a few hours of childbirth will considerably reduce the risk of a further breakdown of the disease, because by these means the diaphragms can be pushed up, the air taking the place of the enlarged uterus and foetus. It should be maintained for a few months and then gradually abandoned.

A further factor to be borne in mind, in assessing the fitness of a patient with pulmonary tuberculosis to continue with a pregnancy, is whether she already has children. Successive pregnancies have an increasingly detrimental effect on the lung condition. As Du Bois has stated: "If a woman with phthisis marries she may bear the first accouchement well, a second with difficulty, a third never.". If she already has a family, termination of a pregnancy will not cause much psychological disturbance. Therefore, in multipara suffering from active pulmonary tuberculosis, termination of pregnancy should be advised.

During the puerpurium and for one or two years afterwards it is wise for the patient to be $\mathrm{X}$-rayed, and her sputum, if any, tested at intervals-say immediately after the birth of the child, and then at $x, 2,3$ and 6 months, and at six-monthly intervals thereafter. The periods between X-rays can be lengthened out in this way because the risk of a breakdown becomes progressively less as time passes.

\section{Quiescent Pulmonary Tuberculosis and Pregnancy}

By the term "quiescent pulmonary tuberculosis" is meant that the disease, as shown by the clinical signs, radiology, and sputa tests, is not active, that it has remained in statu quo for a considerable period, but it is felt that the disease has not completely fibrosed or calcified, and is therefore liable to break down. Such a state of affairs is often found with pulmonary tuberculosis. The patient in this category feels perfectly well and is able to be up and about, undertaking a considerable amount of exercise, but it is not desirable that she should have a child at this stage because of the extra physical strain. In a case where an artificial pneumothorax has been necessary, it is not thought safe to let a pregnancy occur until two or three years after the lung has been allowed to re-expand. The artificial pneumothorax should, if possible, be maintained for three years after its adequate establishment-that is, three years after adhesions, if any, have been divided and a satisfactory collapse has been obtained. The reason for waiting this time after the lung has re-expanded is in order to be sure that the disease has healed. This will be shown by $\mathrm{X}$-rays with more certainty after the lung has come out again. If subsequent serial X-rays show no change, the disease can be regarded as quiescent, and pregnancy can be allowed. In a case where no active interference has been necessary pregnancy should not be permitted until a similar period has elapsed after the disease has become quiescent, during which time the $\mathrm{X}$-rays show no change.

In assessing the fitness of a patient with quiescent tuberculosis to undertake a pregnancy, her general condition is the first consideration. Her weight must be steady, and she must have no symptoms referable to the lung condition. If there is cough and sputum, then it must have remained in the same quantity and been consistently negative for the previous two or three years.

The sedimentation rate is a very valuable guide as to the general condition of the patient. A single sedimentation rate is of value, of course, but serial readings taken at regular intervals 
are of far more value. If the readings are consistently normal, it is a guide that the general condition of the patient is good.

The radiological evidence is a most important consideration. A single film is not sufficient datum on which to assess the fitness of a patient for pregnancy. Serial X-rays taken at regular intervals are essential. The reason for this is that the disease may appear quiescent on a single picture, whereas serial X-rays might reveal a very different state of affairs. Activity on serial $\mathrm{X}$-rays is gauged by the fact that the shadowing alters; this need not necessarily be an increase in the shadowing: a decrease is equally important for this shows that the patient has improved and that she must therefore have had active disease when the first $\mathrm{X}$-ray was taken. If over a number of years the radiological evidence remains unaltered, then pregnancy is permissible. At the same time it is essential that the patient should be $\mathrm{X}$-rayed at regular intervals during the pregnancy-for example, at 3, 5, 7, 8 and 9 months-so that any change can be detected and treated at the earliest possible moment. As in the case of active disease, so with quiescent tuberculosis it is essential for the patient to be X-rayed and have sputa examinations regularly after the birth of the child.

The home conditions of the patient are also important. If the patient is in a position to have adequate domestic help during pregnancy and after the birth of the child, then pregnancy may be safely undertaken within a shorter period of the disease being regarded as quiescent. The importance of adequate rest during and immediately following the pregnancy cannot be overstressed.

\section{Other Considerations}

A further factor is the psychological attitude of the patient. Some women are so keen to have children of their own that they are prepared to rest during the whole pregnancy and to continue resting afterwards. If that is the case, then pregnancy may be permitted even if the patient has active disease, providing the risk to the mother and child is explained to the prospective parents. As a result of the war which has caused the break-up of normal home life, and with the added risk to the husband, this psychological factor is even more important, as illustrated in my third case below.

Breast feeding is contraindicated in the case of a tuberculous patient, whether the diseasea is active or quiescent, because nourishment for the baby has to be supplied by the mother, and this, following on the strain of pregnancy and parturition is unwise. At the same time, in a case of active pulmonary tuberculosis, where there is a positive sputum, it is even more important that the mother should not feed her baby because of the close contact of the mother and child and the risk of infection. With the progress made in artificial feeding the baby comes to no harm, and indeed thrives on it.

While discussing pregnancy in the tuberculous patient, it may not be out of place to mention the following point. It is frequently stated in medical text-books that the tuberculous patient is more highly sexed than the normal person, and it is well known that the pulmonary condition does not affect conception. (Of course, in a case where there is a tuberculous pulmonary lesion associated with a tuberculous salpyngitis, the patient becomes sterile.) The view that the tuberculous patient is more highly sexed than the normal person is probably incorrect, and has arisen because when young people of both sexes are mixing at a sanatorium, leading a physically restricted life, but often feeling very well, it is natural for their interests to tend toward sexual matters. When patients are discharged after institutional treatment, and their interests broaden, this tendency disappears.

\section{Case No. I.-Active Pulmonary Tuberculosis with Termination of Pregnancy.}

Mrs. S., aged 38 , who three years earlier was found to be suffering from pulmonary tuberculosis which had responded well to sanatorium type of treatment, complained of a febrile attack and loss of weight. At that time she was one month pregnant. Her chest was X-rayed, and the film showed active bilateral trouble; her sputum contained tubercle bacilli, and her general condition was poor. In view of the degree of disease present and the fact that she already had several children, termination of the pregnancy was advised. This decision was made easier because she refused to go to sanatorium or to rest adequately. A termination was produced, and her chest condition is improving. (Fig. I.) 


\section{CASE No. II.-Active Pulmonary Tuberculosis in which Pregnancy was Allowed.}

Mrs. M., aged 24, was seen when six months pregnant, with a history of cough, sputum and lassitude. Physical signs suggested a left apical lesion, and these were confirmed radiologically. The disease was unilateral, with cavitation, and her sputum contained tubercle bacilli. At this stage of her pregnancy an abdominal hysterotomy would have been necessary, and it was therefore decided to allow the pregnancy to continue, and to attempt an immediate artificial pneumothorax. This was successfully induced, and an $\mathrm{X}$-ray taken a fortnight later showed an apical adhesion with the cavity still present. Four weeks after the induction this adhesion was divided and the lung completely freed. Six weeks later the cavity could not be seen, and the pregnancy continued normally. No untoward effect was caused at the time of labour by the artificial pneumothorax, and oxygen was not required. The patient's condition since the birth of the child has remained good. (Fig. 2.)

\section{CASE No. III.-Quiescent Pulmonary Tuberculosis and Pregnancy.}

Mrs. B., aged 29, had bilateral pulmonary tuberculosis, with bilateral pneumothoraces, the disease having been present for eight years. She came to see me for a routine examination. Her general condition and chest condition were very good, and I judged the disease to be quiescent. She was very upset because her husband, who was in the army, was likely to be sent abroad. She was afraid that she might not see him again, and keenly wished to become pregnant before he left the country. The risk to her was explained to both of them. She was prepared to rest as much as was necessary, even to the extent of being in bed during the whole of the pregnancy and as long afterwards as might be thought wise. I therefore agreed to the pregnancy. She remained well throughout, and her condition since the birth of her baby nearly a year ago has continued to be good. (Fig. 3.)

\section{CASE No. IV.-Healed Tuberculosis and Pregnancy.}

Mrs. A., aged 40, had bilateral apical pulmonary tuberculosis diagnosed in I932, with a positive sputum. Her condition greatly improved with sanatorium treatment, and remained satisfactory. X-rays were taken at intervals up to January I942, when she became pregnant. Radiologically the disease was judged to be healed and calcified, the patient's sputum did not contain tubercle bacilli, and her general condition was good. It was felt safe to allow the pregnancy to continue, the baby was born in October I942, and the patient has had no relapse. (Fig. 4.)

\section{Summary}

I. In active pulmonary tuberculosis pregnancy should be terminated unless the patient is willing to rest adequately and the disease can be controlled.

2. In quiescent pulmonary tuberculosis pregnancy is permissible if adequate rest is taken.

3. In healed pulmonary tuberculosis there is little risk of the disease breaking down following pregnancy.

4. Breast feeding is contraindicated in all cases.

5. Four cases are cited with $\mathrm{X}$-rays.

\section{REFERENCE}

I. RAYMOND C. COHEN, M.D. Lond., D.P.R., B.M.J. (1943), 11, 775. 